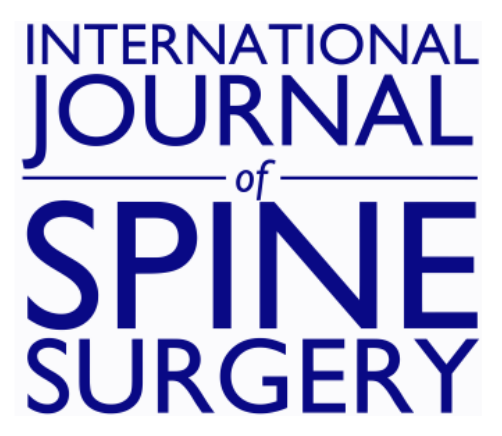

\title{
Comparison of Stand-Alone, Transpsoas Lateral Interbody Fusion at L3-4 and Cranial vs Transforaminal Interbody Fusion at L3-4 and L4-5 for the Treatment of Lumbar Adjacent Segment Disease
}

DEEPTEE JAIN, KUSHAGRA VERMA, JEFFREY MULVIHILL, JUN MIZUTANI, BOBBY TAY, SHANE BURCH and VEDAT DEVIREN

Int J Spine Surg 2018, 12 (4) 469-474

doi: https://doi.org/10.14444/5056

http://ijssurgery.com/content/12/4/469

This information is current as of April 26, 2023.

Email Alerts Receive free email-alerts when new articles cite this article. Sign up at: http://ijssurgery.com/alerts 


\title{
Comparison of Stand-Alone, Transpsoas Lateral Interbody Fusion at L3-4 and Cranial vs Transforaminal Interbody Fusion at L3-4 and L4-5 for the Treatment of Lumbar Adjacent Segment Disease
}

\author{
DEEPTEE JAIN, MD, KUSHAGRA VERMA, MD, JEFFREY MULVIHILL, MD, JUN MIZUTANI, MD, \\ BOBBY TAY, MD, SHANE BURCH, MD, VEDAT DEVIREN, MD \\ Department of Orthopaedic Surgery, University of California, San Francisco, San Francisco, California
}

\begin{abstract}
Study Design: Retrospective cohort study.

Objective: To compare outcomes and complications of stand-alone minimally invasive lateral interbody fusion (LIF) vs revision posterior surgery for the treatment of lumbar adjacent segment disease.

Methods: Adults who underwent LIF or transforaminal lumbar interbody fusion (TLIF) for adjacent segment disease were compared. Exclusion criteria: $>$ grade 1 spondylolisthesis, posterior approach after LIF, and L5/S1 surgery. Patient demographics, estimated blood loss, hospital length of stay, complications, reoperations, health-related quality of life measures, and radiographs were examined. Data were analyzed with the $\chi^{2}$, Wilcoxon signed rank, and MannWhitney $U$ tests.

Results: A total of 17 LIF and 16 TLIF patients were included. Demographics were similar. Follow up was similar (LIF: $22.9 \pm 11.8$ months vs TLIF: $22.0 \pm 4.6$ months; $P=.86$ ). The LIF patients had significantly less blood loss (LIF: $36 \pm 16 \mathrm{~mL}$ vs TLIF: $700 \pm 767 \mathrm{~mL} ; P<.001$ ) and shorter length of stay (LIF: $2.6 \pm 2.9$ days vs TLIF: $3.3 \pm 0.9$ days; $P=.001)$. There were no intraoperative complications. Revision rate was 4 of 17 in LIF and 3 of 16 in TLIF $(P=.73)$. Baseline health-related quality of life and radiographic measurements were similar. In both groups, back and leg pain scores significantly improved, and in LIF, the Owestry Disability Index, and EuroQol-5D significantly improved. The LIF had a significant increase in intervertebral height (LIF: $4.8 \pm 2.9 \mathrm{~mm}, P<.001$, TLIF: $1.3 \pm 3.4 \mathrm{~mm}, P=.37)$, which was significantly greater for LIF than TLIF $(P=.002)$. Similarly, LIF had a significant increase in segmental lordosis (LIF: $5.6^{\circ} \pm 4.9^{\circ}, P<.001$, TLIF: $3.6^{\circ} \pm 8.6^{\circ}, P=.16$ ), which was not significantly different between groups.
\end{abstract}

Conclusions: Patients with adjacent segment disease may receive significant benefit from stand-alone LIF or TLIF. The LIF offers advantages of less blood loss and a shorter hospital stay.

Level of Evidence: 3

Minimally Invasive Surgery

Keywords: lateral interbody fusion, minimally invasive, adjacent segment disease, transforaminal lumbar interbody fusion

\section{INTRODUCTION}

Adjacent segment disease (ASD), characterized by degeneration and/or stenosis, is a common phenomenon seen in patients who have undergone prior lumbar fusion surgery. The rate of ASD has been estimated radiographically and clinically to be over $40 \%$ and over $30 \%$, respectively, at the 5 -year follow-up. ${ }^{1}$ This process may be a result of increased biomechanical forces at the levels either above or below the fusion, or it might be influenced by natural history in a patient who has already demonstrated degenerative changes requiring the index operation. Patients may present with axial pain from disc or facet degeneration or with symptoms of neural compression such as neurogenic claudication, radiculopathy, or both.

Treatment for ASD has historically involved extension of the fusion with a posterior approach, with direct decompression of the neural elements if indicated. Revision surgery, however, has the disadvantages of longer operative time, greater blood loss, and longer hospital stays as well as increased risk of intraoperative dural tears. ${ }^{2,3}$ In 
addition, exposure and instrumentation around the adjacent lumbar facets in standard open surgery may predispose to accelerated adjacent segment degeneration. Alternatively, more novel minimally invasive approaches have been gaining favor, especially the minimally invasive lateral approach developed by Pimenta. ${ }^{4}$ The lateral approach allows the surgeon to achieve a robust fusion through the anterior column and relies on the theory of indirect decompression of the neural elements. ${ }^{5}$ Although initially there existed concerns about the success of fusion given the stiff adjacent construct, more recent evidence has shown that stand-alone minimally invasive lateral interbody fusion (LIF) can be successful in treating ASD. In a case series of 21 patients, Wang et al demonstrated improved pain scores, no complications, and 1 revision surgery for direct decompression with this approach. ${ }^{6}$ This study, however, did not review patient-reported outcome measures, and furthermore, as a case series, was level 4 evidence. Here, we present a retrospective cohort study aiming to compare patients who underwent stand-alone LIF vs a more traditional posterior approach, open transforaminal lumbar interbody fusion (TLIF), including clinical as well radiographic analysis. We hypothesize that LIF results in improved clinical and radiographic outcomes as compared with TLIF.

\section{METHODS}

This was a retrospective cohort study. The following is presented as according to the Strengthening the Reporting of Observational Studies in Epidemiology statement.

After institutional review board approval was obtained at our institution, adults (age $>18$ years) who underwent LIF or TLIF for single-level ASD between 2010 and 2015 at a single institution were compared. Patients who had $>$ grade 1 degenerative spondylolisthesis; underwent a planned, staged posterior approach after LIF; or underwent L5/ S1-level surgery were excluded. Patients with less than 1 year of follow-up were also excluded. The choice to undergo LIF or TLIF was made by the treating surgeon.

Data were collected retrospectively from chart review. Demographics collected included age, gender, American Society of Anesthesiologists score, smoking status, and body mass index. Operative and hospital details collected included estimated blood loss and hospital length of stay. Health- related quality of life (HRQoL) scores including visual analog scale (VAS) for back and leg pain, the Owestry Disability Index (ODI), and the EuroQol5D (EQ-5D) were collected preoperatively and postoperatively at the date of final follow-up between 1 and 2 years. Radiographs were analyzed preoperatively and postoperatively for segmental lordosis and intervertebral disc height. All measurements were made by 2 authors on 2 separate occasions. Segmental lordosis was measured as the Cobb angle of the superior end plate of the cranial vertebra and the inferior end plate of the caudal vertebra on a lateral x-ray at the operative level. Intervertebral disc height was measured as the distance from the midpoint of the inferior endplate of the cranial vertebra to the midpoint of the superior end plate of the caudal vertebra on a lateral $x$-ray at the operative level. The intraobserver reliability for segmental lordosis was .83 and .71 and for intervertebral disc height was .74 and .82 . The interobserver reliability for segmental lordosis was .68 and for intervertebral disc height was .65 . Finally, all perioperative and postoperative complications and revisions were diligently followed.

Data were described as mean and standard deviation. Continuous demographic and operative data were compared between groups using MannWhitney $U$ tests. Discrete data were compared between groups using $\chi^{2}$. The HRQoL measures and radiographic measurements were compared within groups using Wilcoxon signed rank tests and between groups using Mann-Whitney $U$ tests. All analyses were performed using Stata, version 13.1 (StataCorp, College Station, TX).

\section{RESULTS}

A total of 17 patients in the LIF group and 16 patients in the TLIF group met the inclusion/ exclusion criteria. Demographic data are presented in Table 1. There were no significant demographic differences between groups. Average follow-up was similar between groups (LIF: $22.9 \pm 11.8$ vs TLIF: $22.0 \pm 4.6$ months; $P=.86$ ).

There was significantly more bone morphogenetic protein 2 used in the LIF group (LIF: 5/17 vs TLIF $0 / 16 ; P<.001)$. The operative levels between groups were significantly different (LIF: 1 at L1-2, 3 at L2-3, 13 at L3-4, 0 at L4-5 vs TLIF: 0 at L1-2, 0 at L2-3, 8 at L3-4, 8 at L4-5; $P=.004)$. There were no intraoperative complications in either group. Estimated blood loss was significantly less in the 
Table 1. Patient demographics (all values are presented as the mean and standard deviation).

\begin{tabular}{lccc}
\hline Characteristic & LIF, $\mathbf{n}=\mathbf{1 7}$ & TLIF, $\mathbf{n}=\mathbf{1 6}$ & $\boldsymbol{P}$ \\
\hline Age, y & $63.4 \pm 12.9$ & $57.4 \pm 9.5$ & .13 \\
Gender & 8 & 5 & .86 \\
$\quad$ Male & 9 & 11 & \\
$\quad$ Female & 1 & 3 & .26 \\
Smoking status & 16 & 13 & \\
$\quad$ Smoker & $2.0 \pm 0.4$ & $2.3 \pm 0.5$ & .13 \\
$\quad$ Nonsmoker & $28.9 \pm 4.1$ & $33.2 \pm 9.3$ & .20 \\
ASA score & $22.9 \pm 11.8$ & $22.0 \pm 4.6$ & .86 \\
BMI, kg/m & & & \\
Follow-up, mo & & & \\
\hline
\end{tabular}

Abbreviations: ASA, American Society of Anesthesiologists; BMI, body mas index; LIF, lateral interbody fusion; TLIF, transforaminal lumbar interbody fusion.

LIF group (LIF: $36 \pm 16 \mathrm{~mL}$ vs TLIF: $700 \pm 767$ $\mathrm{mL} ; P<.001)$. Length of stay was significantly shorter in the LIF group (LIF: $2.6 \pm 2.9$ days vs TLIF: $3.3 \pm 0.9$ days; $P=.001)$. There were no perioperative complications in either group. There was no new numbness or weakness indicative of a lumbar plexopathy seen in the LIF group as a result of the procedure.

Baseline clinical outcome scores were similar, except for worse VAS back pain in the TLIF group $(P=.028$; Table 2). Postoperative clinical outcome scores were also similar, except again for worse VAS back pain in the TLIF group $(P=.021)$. There were no significant differences between groups in the change-of-pain scores or HRQoL scores. In both groups, back and leg pain significantly improved (LIF: change in VAS back pain $-1.8 \pm 3.0$, $P=.049$, change in VAS leg pain $-2.4 \pm 2.7$, $P=.041$, TLIF: change in VAS back pain $-2.2 \pm 3.3, P=.028$, change in VAS leg pain $-2.6 \pm 2.7, P=.002)$. The ODI and EQ-5D significantly improved in the LIF group and trended toward improvement in the TLIF group (LIF: change in ODI $-14.4 \pm 16.9, P=.028$, change in EQ-5D $0.21 \pm 0.23, P=.041$, TLIF: change in ODI $-7.5 \pm 21.5, \quad P=0.31$, change in EQ-5D $0.15 \pm 0.37, P=.14)$.

Baseline radiographic measurements were similar (Table 2). The LIF had significant increases in intervertebral disc height (LIF: $4.8 \pm 2.9 \mathrm{~mm}$, $P<.001$, TLIF: $1.3 \pm 3.4 \mathrm{~mm}, P=.37$ ), which was significantly greater for LIF than TLIF $(P=.002)$. Similarly, LIF had a significant increase in segmental lordosis (LIF: $5.6^{\circ} \pm 4.9^{\circ}, P<.001$, TLIF: $\left.3.6^{\circ} \pm 8.6^{\circ}, P=.16\right)$, which trended toward being greater for LIF than TLIF $(P=.23)$.

Four patients $(24 \%)$ in the LIF group and 3 patients $(19 \%)$ in the TLIF group underwent
Table 2. Clinical and radiographic outcomes (all values are presented as the mean and standard deviation).

\begin{tabular}{|c|c|c|c|}
\hline & LIF & TLIF & $P$ \\
\hline \multicolumn{4}{|c|}{ VAS back pain } \\
\hline Pre-op & $5.1 \pm 3.1$ & $8.3 \pm 1.7$ & .028 \\
\hline Post-op & $3.3 \pm 2.5$ & $6.1 \pm 3.1$ & .021 \\
\hline Change & $-1.8 \pm 3.0$ & $-2.2 \pm 3.3$ & .86 \\
\hline$P$ & .049 & .028 & \\
\hline \multicolumn{4}{|c|}{ VAS leg pain } \\
\hline Pre-op & $4.0 \pm 2.4$ & $5.7 \pm 2.1$ & .078 \\
\hline Post-op & $1.6 \pm 1.9$ & $3.1 \pm 2.3$ & .071 \\
\hline Change & $-2.4 \pm 2.7$ & $-2.6 \pm 2.7$ & .82 \\
\hline$P$ & .041 & .002 & \\
\hline \multicolumn{4}{|l|}{ ODI } \\
\hline Pre-op & $47.5 \pm 16.2$ & $54.2 \pm 15.8$ & .35 \\
\hline Post-op & $33.2 \pm 20.9$ & $46.8 \pm 18.4$ & .082 \\
\hline Change & $-14.4 \pm 16.9$ & $-7.5 \pm 21.5$ & .50 \\
\hline$P$ & .028 & .31 & \\
\hline \multicolumn{4}{|c|}{ EQ-5D Utility Score } \\
\hline Pre-op & $0.49 \pm 0.19$ & $0.42 \pm 0.22$ & .47 \\
\hline Post-op & $0.70 \pm 0.20$ & $0.57 \pm 0.23$ & .059 \\
\hline Change & $0.21 \pm 0.23$ & $0.15 \pm 0.37$ & .81 \\
\hline$P$ & .041 & .14 & \\
\hline \multicolumn{4}{|c|}{ Segmental lordosis } \\
\hline Pre-op & $12.6 \pm 7.4$ & $13.1 \pm 5.3$ & .66 \\
\hline Post-op & $18.2 \pm 8.4$ & $16.7 \pm 7.3$ & .57 \\
\hline Change & $5.6 \pm 4.9$ & $3.6 \pm 8.6$ & .23 \\
\hline$P$ & $<.001$ & .16 & \\
\hline \multicolumn{4}{|c|}{ Intervertebral disc height } \\
\hline Pre-op & $6.5 \pm 2.7$ & $8.0 \pm 3.2$ & .11 \\
\hline Post-op & $11.4 \pm 2.6$ & $9.3 \pm 2.7$ & .032 \\
\hline Change & $4.8 \pm 2.9$ & $1.3 \pm 3.4$ & .002 \\
\hline$p$ & $<0.001$ & 0.37 & \\
\hline
\end{tabular}

Abbreviations: EQ, EuroQuol; LIF, lateral interbody fusion; ODI, Oswestry Disability Index; TLIF, transforaminal lumbar interbody fusion; VAS, visual analog scale.

revision surgery. The rate of reoperation was not significantly different between groups $(P=.73)$. In the LIF group, all patients who underwent revision had posterior decompression with instrumentation. The indications were as follows: 2 for persistent radiculopathy, 1 for subsidence and development of radiculopathy, and 1 for implant subluxation. In the TLIF group, 1 patient underwent TLIF and extension of posterior fusion for ASD at the level above, 1 patient underwent anterior LIF and extension of posterior fusion for ASD at the level below, and 1 patient underwent revision decompression for recurrent radiculopathy.

A case example of a patient who underwent LIF is presented in Figures 1 and 2.

\section{DISCUSSION}

In this retrospective cohort study, we compared stand-alone LIF and TLIF for the treatment of lumbar ASD. The findings demonstrate that both LIF and TLIF can be used to treat lumbar ASD safely and effectively with good clinical outcomes at the 1- to 2-year follow-up, and LIF has the added 

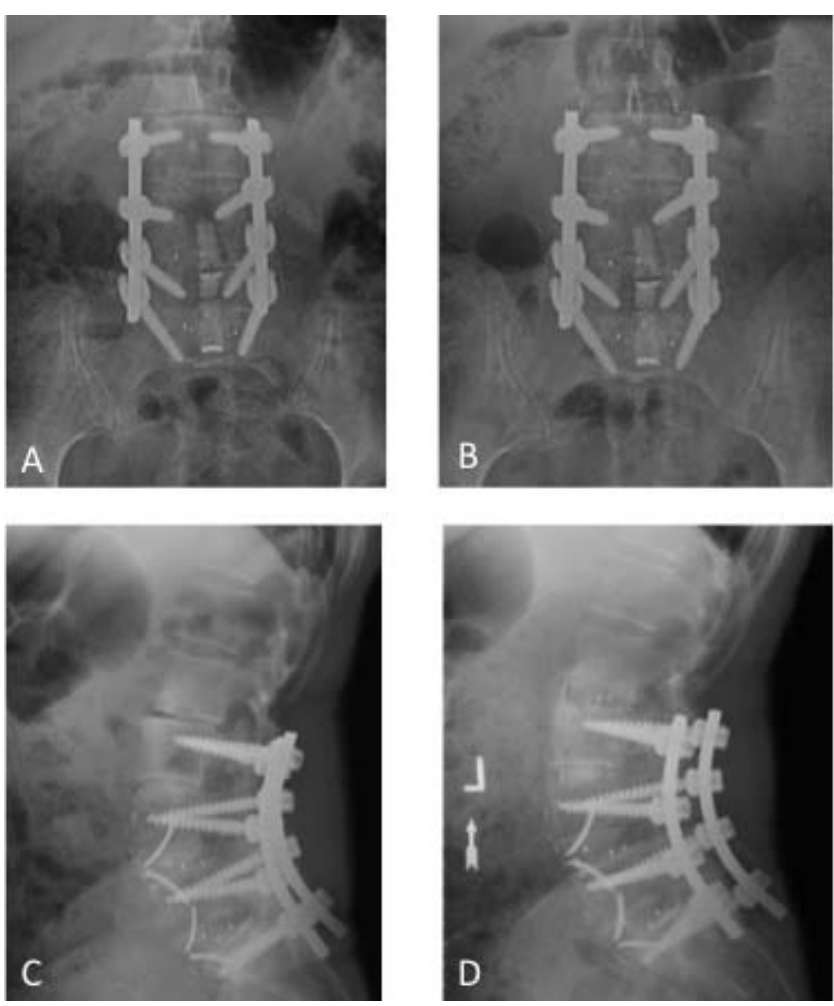

Figure 1. Case example of lumbar interbody fusion (LIF). Preoperative anterior-posterior $(\mathrm{A})$ and lateral $(\mathrm{C})$ radiographs demonstrate disc collapse at L2/3 secondary to adjacent segment disease (ASD). Postoperative anteriorposterior (B) and lateral (D) radiographs demonstrate restoration of disc height.

benefits of less estimated blood loss and shorter length of stay.

Patients who underwent LIF had significant improvements in back and leg pain, ODI, and EQ5D. Patients who underwent TLIF also had significant improvement in pain scores. It should be noted that although we were unable to demonstrate statistically significant improvement in ODI and EQ-5D in the TLIF group, it is likely that these findings represent a type II error secondary to inadequate power, and therefore, in reality, it is likely that TLIF does indeed improve these measures. The improvements seen in the LIF group were similar to those seen in the TLIF group, as demonstrated by similar absolute value changes in pain scores and HRQoLs. Thus, either approach may be an effective option for treating lumbar ASD.

The LIF patients had significantly less blood loss and shorter length of stay than TLIF patients. These findings in the LIF group are consistent with those demonstrated in the case series by Wang et al as well as other studies. ${ }^{6-8}$ Here, we prove that the LIF approach, as compared with a revision posterior approach, has the added benefit of avoiding the
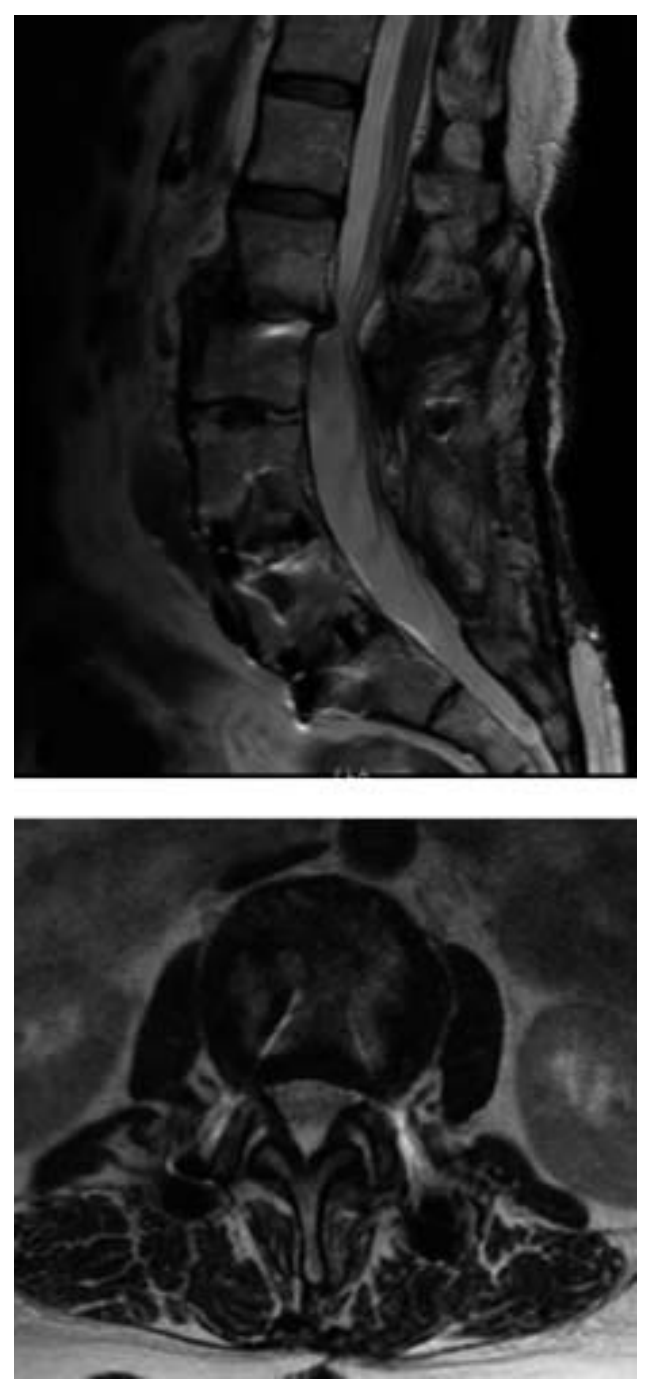

Figure 2. Preoperative magnetic resonance imaging, T2 weighted midsagittal cut (above) and axial cut at L2/3 (below) demonstrate disc degeneration, bilateral lateral recess stenosis, and central stenosis at L2/3.

morbidity associated with increased blood loss and also aids in a faster recovery from surgery.

Patients who underwent LIF also demonstrated a significant improvement in intervertebral disc height, which was significantly more than that achieved with TLIF. This was as expected; the LIF approach allows direct access to the disc space and the ability to place a large cage, more so than traditional posterior open approaches. ${ }^{9,10}$ It is this increase in disc height that allows for indirect neural compression.

Patients who underwent LIF had a significant improvement in segmental lordosis. This improvement is consistent with that seen in multiple previous studies. ${ }^{11}$ Restoring lumbar lordosis is of paramount importance; more recently, the literature has presented increasing emphasis on the role of the 
malaligned spine after short segment lumbar fixation in the development of ASD. ${ }^{12}$

In addition, the LIF approach has the benefit of avoiding disruption of the posterior tension band as well as anterior and posterior longitudinal ligaments. Disruption of these structures has also been hypothesized to be a risk factor for ASD. ${ }^{1,13}$ Consistent with this theory, in our study, we demonstrated that there were no revisions for recurrent ASD in the LIF group but 2 revisions for ASD in the TLIF group, suggesting that LIF may be protective against ASD as compared with a traditional posterior approach. Patients undergoing surgery for ASD have already demonstrated a propensity for degeneration with their own unique biologic and environmental milieu, whether it be a natural progression of their disease process or iatrogenic; therefore, preventing ASD in the future is of special concern when treating the current ASD.

We think it important that there was approximately a $25 \%$ revision rate in patients undergoing LIF. This may provide insight into some of the limitations of the LIF approach. As mentioned, the minimally invasive lateral interbody approach relies on indirect decompression of the neural elements, elevating the vertebral body to distract and relieve compression by opening up the central canal and the foramen. ${ }^{5}$ This may be inadequate in patients with severe compression. Two patients in the LIF group required a revision posterior approach for further decompression, whereas none of the patients in the TLIF group required such a procedure. This was also noted in the case series by Wang et al. ${ }^{6}$

Another concern with using of the LIF approach regards implant stability. Whereas LIF has been performed successfully in patients with spondylolisthesis and degenerative scoliosis, ${ }^{13,14}$ in this study, we did limit the use of stand-alone LIF to those patients without significant coronal or sagittal imbalance or evidence of dynamic spondylolisthesis. Nonetheless, 1 of the patients in the LIF group in this study necessitated revision for subluxation. Cage settling has been previously seen both with stand-alone cages and those that are augmented posteriorly, and it is unclear whether posterior fixation can prevent this problem. ${ }^{7}$ Furthermore, the instability seen in stand-alone LIF can be a cause of residual radicular symptoms already mentioned. When performing LIF, it is important to select patients with good bone density and also, technically, to preserve the integrity of the end plates.
Finally, no patients in the LIF group in this study underwent revision surgery for pseudarthrosis. Previous studies have demonstrated $100 \%$ fusion rate in patients undergoing stand-alone LIF as examined by computed tomography scan and another 2 as examined by need for revision surgery. ${ }^{6,7,15}$ In this study, we did not have computed tomography scans on all patients and were therefore unable to radiographically assess fusion rates. As with other approaches for interbody fusion, LIF promotes fusion on the compression side of the construct, as opposed to the tension side of the construct, and fusion rates are likely similar to other interbody approaches.

Whereas there is a high revision rate with the LIF approach, notably, the revision surgery is the same as the initial alternative for treating ASD - a revision posterior approach with extension of the fusion. Furthermore, we were unable to demonstrate a difference in the revision rate as compared with TLIF. As such, in the appropriately selected patient, it is reasonable to offer LIF, with hopes of a faster recovery and avoidance of complications associated with revision posterior surgery, with the understanding that $25 \%$ of patients might require a second surgery. This decision requires an informed discussion with the patient.

Other limitations of this study include its small number of patients, which makes it difficult to detect differences between the 2 groups, specifically in terms of revision rates. Another limitation includes the use of recombinant human bone morphogenetic protein 2 in the LIF group but not the TLIF group. Bone morphogenetic protein is US Food and Drug Administration-approved for use in anterior LIF; thus far, there have been no reports in this study or others of any complication associated with its use in LIF. ${ }^{6,16}$ It is, however, contraindicated in TLIF due to the high incidence of radiculitis. ${ }^{17}$

Finally, there is likely a selection bias in selecting stand-alone LIF vs TLIF with revision posterior decompression and fusion for the treatment approach for 2 main reasons. First, as previously mentioned, given that LIF depends on indirect decompression, we suggest LIF for patients with less severe stenosis and TLIF for those with more severe stenosis. This may be evident on imaging or clinically. If the patient has a severe lateral recess or neuroforaminal stenosis on imaging or has evidence of fixed radiculopathy that does not improve with positioning, LIF is less likely to be 
successful than TLIF with a direct decompression. Second, the selection bias is also reflected in the fact that the levels treated between the 2 groups were significantly different, with no LIF performed at L45 and no TLIF performed cranial to L3-4. This represents technical safety challenges unique to each procedure. At the L4-5 level, the lumbar plexus runs more anteriorly within the psoas, thus making the safe zone much narrower to perform a LIF. At the more cranial levels, the cord and conus do not tolerate manipulation; as a result, a much wider decompression is required to safely perform a TLIF. Furthermore, caudal segments experience more motion and biomechanical stresses as compared with more cranial segments. Thus, ASD caudal to a fusion should be treated with TLIF due to concerns regarding implant stability with a stand-alone LIF.

In conclusion, this retrospective cohort study demonstrates that good outcomes can be achieved using stand-alone minimally invasive lateral interbody fusion to treat ASD, with lower blood loss and shorter length of hospital stay than a traditional posterior approach. With a $25 \%$ revision rate, this procedure should be performed in an appropriately selected patient with good bone quality and without severe neural compression.

\section{REFERENCES}

1. Cheh G, Bridwell KH, Lenke LG, et al. Adjacent segment disease following lumbar/thoracolumbar fusion with pedicle screw instrumentation: a minimum 5-year follow-up. Spine. 2007;32(20):2253-2257.

2. Parker SL, Shau DN, Mendenhall SK, McGirt MJ. Factors influencing 2-year health care costs in patients undergoing revision lumbar fusion procedures: clinical article. J Neurosurg Spine. 2012;16(4):323-328.

3. Smorgick Y, Baker KC, Bachison CC, Herkowitz HN, Montgomery DM, Fischgrund JS. Hidden blood loss during posterior spine fusion surgery. Spine J. 2013;13(8):877-881.

4. Ozgur BM, Aryan HE, Pimenta L, Taylor WR. Extreme lateral interbody fusion (XLIF): a novel surgical technique for anterior lumbar interbody fusion. Spine J. 2006;6(4):435-443.

5. Kepler CK, Sharma AK, Huang RC, et al. Indirect foraminal decompression after lateral transpsoas interbody fusion: clinical article. J Neurosurg Spine. 2012;16(4):329-333.

6. Wang MY, Vasudevan R, Mindea SA. Minimally invasive lateral interbody fusion for the treatment of rostral adjacentsegment lumbar degenerative stenosis without supplemental pedicle screw fixation: clinical article. J Neurosurg Spine. 2014;21(6):861-866.

7. Na YC, Lee HS, Shin DA, Ha Y, Kim KN, Yoon DH. Initial clinical outcomes of minimally invasive lateral lumbar interbody fusion in degenerative lumbar disease: a preliminary report on the experience of a single institution with 30 cases. Korean J Spine. 2012;9(3):187-192.
8. Palejwala SK, Sheen WA, Walter CM, Dunn JH, Baaj AA. Minimally invasive lateral transpsoas interbody fusion using a stand-alone construct for the treatment of adjacent segment disease of the lumbar spine: review of the literature and report of three cases. Clin Neurol Neurosurg. 2014;124(Sep):9096.

9. Elowitz E, Yanni D, Chwajol M, Starke R, Perin N. Evaluation of indirect decompression of the lumbar spinal canal following minimally invasive lateral transpsoas interbody fusion: radiographic and outcome analysis. Min Invasive Neurosurg. 2011;54(5-6):201-206.

10. Oliveira L, Marchi L, Coutinho E, Pimenta L. A radiographic assessment of the ability of the extreme lateral interbody fusion procedure to indirectly decompress the neural elements. Spine. 2010;35(26S):S331-S337.

11. Uribe JS, Myhre SL, Youssef JA. Preservation or restoration of segmental and regional spinal lordosis using minimally invasive interbody fusion techniques in degenerative lumbar conditions. Spine. 2016;41(1):S50-S58.

12. Rothenfluh DA, Müller DA, Rothenfluh E, Min K. Pelvic incidence-lumbar lordosis mismatch predisposes to adjacent segment disease after lumbar spinal fusion. Eur Spine J. 2015;24(6):1251-1258.

13. Helgeson MD, Bevevino AJ, Hilibrand AS. Update on the evidence for adjacent segment degeneration and disease. Spine J. 2013;13(3):342-351.

14. Isaacs RE, Hyde J, Goodrich JA, Rodgers WB, Phillips FM. A prospective, nonrandomized, multicenter evaluation of extreme lateral interbody fusion for the treatment of adult degenerative scoliosis: perioperative outcomes and complications. Spine. 2010;35(26S):S322-S330.

15. Khajavi K, Shen A, Lagina M, Hutchison A. Comparison of clinical outcomes following minimally invasive lateral interbody fusion stratified by preoperative diagnosis. Eur Spine J. 2015;24(3):322-330.

16. Burkus JK, Transfeldt EE, Kitchel SH, Watkins RG, Balderston RA. Clinical and radiographic outcomes of anterior lumbar interbody fusion using recombinant human bone morphogenetic protein-2. Spine. 2002;27(21):2396-2408.

17. Mindea SA, Shih P, Song JK. Recombinant human bone morphogenetic protein-2-induced radiculitis in elective minimally invasive transforaminal lumbar interbody fusions: a series review. Spine. 2009;34(14):1480-1484.

Disclosures and COI: The authors received no funding for this study and report no conflicts of interest.

Corresponding Author: Deeptee Jain, MD, 500 Parnassus Ave, MU West 321, Box 0728, San Francisco, CA 94143. Phone: (414) 364-7762; Fax: (415) 476-1304; Email: jaind@orthosurg.ucsf.edu.

Published 31 August 2018

This manuscript is generously published free of charge by ISASS, the International Society for the Advancement of Spine Surgery. Copyright (C) 2018 ISASS. To see more or order reprints or permissions, see http://ijssurgery.com. 